\title{
Study on Application of Magnetic Shape Memory Alloys to Vibration Control of Structures
}

\author{
Kangning Liu ${ }^{{ }^{*}}$, Shiming Zhang and Xiaohui Peng ${ }^{\mathrm{c}}$ \\ School of Civil Engineering, Xijing University, Xi'an 710123, China \\ a1971576166@qq.com,,bzhangshimin@aliyun.com,c565938711@qq.com
}

\begin{abstract}
Keywords: Vibration and wave; Magnetic shape memory alloy (MSMA); Vibration control; Characteristic of magnetic control; Actuator
\end{abstract}

\begin{abstract}
Magnetic shape memory alloy (MSMA) is a new intelligent material that can produce large stain in magnetic field, and mean while has the shape memory function. Using these characteristics of MSMA, one can fabricate intelligent actuators for vibration control of structures. At first, the mechanism of deformation of MSMA in magnetic field and it $\mathrm{s}$ characteristics of magnetic control are introduced in this paper. On this base, two new MSMA actuators, which may be applied to vibration control of structures, are proposed. Their working principle is analyzed and the design method is given. Finally, the reciprocal characteristics of MSMA, key technology and its solution method of the MSMA self-sensing actuators are described. This study may set a foundation for MSMA's application in civil engineering.
\end{abstract}

\section{Introductions}

It is one of the hot spots to using smart materials for structural vibration control in the study of civil engineering. In recent years, there are many intelligent materials by researching and exploiting can be used for making control devices, mainly have the electrorheological fluids or the magnetorheological fluids, shape memory materials, piezoelectric materials and magnetostrictive materials, etc. Using the intelligent drive materials can make active control drive devices and variable damping devices which are adjusted by electricity, magnetism and temperature, and it will be a new way of structural vibration control because it powerful output, small energy and quick response[1].

Magnetic Shape Memory Alloy(MSMA) is a new SMA materials according to research and development in recent years. It was discovered by professor Uilakko in 1996 in the process of observing the Heusler type alloy Ni2MnGa's performance under the magnetic filed effect[2]. MSMA not only have the characters of normal SMA materials such as temperature shape memory effect, phase change pseudo elastic performance, variable damping small resistance and so on, it also has the magnetic shape memory alley controled by magnetic. The magnetic shape memory effect of MSMA can gets phase change driving force via plus a magnetic field which can induce martensite phase transformation or ferromagnetic phase transformation because of the magnetic phase transformation strain it produced. The magnetic matensite phase transformation of MSMA not only has a powerful magnetic strain, but the action of the magnetic phase is in the instantaneous. so, it's actuation ferquency is equalled to piezoelectric materials but far more than normal SMA materials, it can meet the requirements of the general automatic control system to drive's dynamic response speed[3]. At present, the Heu-sler type alloy has been exploited and put into engineering application, for example, the magneto recoverable strain of Ni2MnGa MSMA can reach 6\%-10\% in normal temperature. its response frequency can also surpass $100 \mathrm{~Hz}[4-5]$. At the same time, the MSMA can come up internal fiction of the matensite phase transformation under the magnetic field and the stress, and induce the should stress and hysteresis phenomenon[6-7]. The additional damping produced by the hysteresis phenomenon has inhibiting effects to the engineering structure vibration. The MSMA material showed characters, such as low martensitic phase transformation, the large recoverable strain, high response frequency and phase transition of internal friction, induced by the magnetic field. 
This thesis introduce the magnetic control character of MSMA material and deformation mechanism under the effect of magnetic field at first, and then put forward two new MSMA drivers that can be applied in the structure vibration control, and analyze its working principle and design method. After that, in the basis of exposition the inverse characteristic of MSMA, come up with the key technology and solutions to make the MSMA sensing actuator.

\section{The Deformation Mechanism of the MSMA Materials}

MSMA material is a shape memory alloy drived by magnetic field, its shape memory effect and deformation mechanism is particular. Ni2MnGa type alloy is the most thorough study magnetic control shape memory alloy materials at present study. Magnetically-controlled shape memory effect (MSM) of MSMA is the transformation of the martensitic transformation of the austenitic phase into the martensite phase and the martensitic transformation of the martensite phase After changing the shape, not only by heating but also by removing the magnetic field by the reverse phase transformation back to the original austenite phase, making the material can be completely restored to the deformation before the shape and volume[8].

The strain generated by MSMA (such as $\mathrm{Ni} 2 \mathrm{MnGa}$ ) martensitic transformation is mainly achieved through the movement of the martensitic interface. The movement of the Martensitic variants's interface needed to lower energy and don't require to destroy the atomic bonds, but can produce larger macro strain through the movement of the variation of the interface to realize the variation preferred orientation. When the MSMA is subjected to an the external magnetic field, the magnetic field can induce the MSMA martensitic variant to move or rotate, and the MSMA easy magnetization direction and the magnetization direction of the MSMA are tends to be uniform, and result in macroscopic strain.

The magnetic drive memory mechanism of $\mathrm{Ni} 2 \mathrm{MnGa}$ alloy lies in the reorientation of the magnetic field-induced twinning. Under action of outside magnetic field $\mathrm{H}$, the magnetocrystalline anisotropy acts as a driving force to rotate the twin variant $\delta$, which makes the direction of the Ni2MnGa's easy magnetization and the direction of applied magnetic field tend to be consistent. Since the direction of easy magnetization in the initial twin variant $\delta$ is not consistent with the direction of the applied magnetic field, when the magnetocrystalline anisotropy energy is larger than the sum of the twin variants boundary energy and the energy required for the external work of the twin variant $\delta$, the twin variants are tilted to produce a transition from the delta variants to the gamma variants, i.e., $\delta^{*} \gamma$. The easy magnetization direction $\mathrm{M}$ in the variant coincides with the external magnetic field direction $\mathrm{H}$, and the total energy of the system decreases. Due to the drive of the magnetocrystalline anisotropic energy, the twins variant $\gamma$ in which easy to magnetization direction and external magnetic field direction is consistent will gradually grow, while the twin variant $\delta$ are gradually reduce, and leading to the twin variant boundary movement, resulting in macro-deformation[9], as given in Fig. 1.
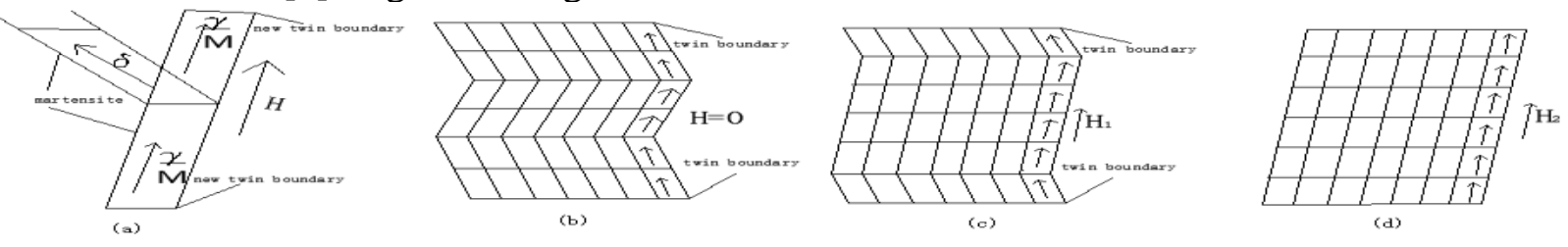

Figure 1. Schematic Diagram of Preferential Orientation of Twins Induced by Magnetic Field

\section{The MSMA Magnetic Control Characteristics and Mechanical Properties}

The MSMA is a kind of new intelligent material, for it can be applied to structural vibration control well, must deeply understand its magnetic control feature, comprehensive the research of scholars in the past. The main magnetic control characteristics are as follows [8,10-15].

(1) Only along the MSMA's close to vertical direction of easy axis (around $110^{\circ}$ or $290^{\circ}$ )impose a magnetic field, can gain maximum deformation. Under the same magnetic field direction, the 
greater magnetic field intensity (or magnetic flux density), the greater the deformation produced by MSMA. The field winding be used to produce magnetic can be either dc, also can lead to the alternating current.

(2) The MSMA produced a deformation under the action of a magnetic field, can't automatically return to its original shape after the magnetic field removal, but vibration control of the structure need the MSMA to round-deformation. According to study, there are two mainly methods to recover deformation. First is applying a certain intensity of magnetic field along the direction of the specimen deformation, the method can make its restore to its original shape. Second is exerting a large enough opposing force with a spring on elongate direction of the MSMA element, the method can make the shape of element restored the previous shape.

(3)Although the shape of the MSMA element can be restored by exerting pressure, but the elongation will decrease with the increase of prestressing force, and is a linear relationship. It creates a contradiction for the size of the pre-pressure force. By preloading force, MSMA cannot restore deformation. And the prestressing force is too large, MSMA's initial deformation is too small, that cannot take full advantage of the deformation of the MSMA material. In order to the MSMA actuator work in the best condition, need to optimize the control of the MSMA preloading force.

(4) MSMA material permeability is low, so when adding the magnetic field should design a form of closed magnetic circuit, avoid the happening of the magnetic flux leakage phenomenon.

\section{The Application of MSMA in the Control of the Structural Vibration Control}

In research field of the intelligent material structure system and active control of structural vibration and so on, an important branch is to study the actuator with high performance, which developed an intelligent actuators with higher strain, wider frequency range and greater output on the basis of existing technology. Actuator can change directly the signals output by controller into strain or displacement of the structure, and can change the shape of the structure, rigidity, location, natural frequency, damping and other mechanical properties. MSMA has the memory effect of magnetic shape controlled by magnetic field, and can be gained magnetic strain and phase transformation driving force through additional magnetic field, and the action is conducted in instantaneous, so that it can create high-performance intelligent actuator and is applied to structural vibration control. In the paper, using magnetic control characteristic of the MSMA materials, preliminary designing one actuators that can be used for structural vibration control, are presented as follows:

\section{The Linear Displacement Actuator}

Working Principle of MSMA Linear Displacement Actuator. The magnetic field generated by electromagnet, the magnetic field's magnitude and direction could be controlled by adjusting the electromagnet field winding current control. In order to make the small magnetic field produce a larger deformation, the direction of the additional magnetic field and the direction of MSMA material producing deformation should be horizontal vertical. When the MSMA element in the magnetic field reaches a certain value, the element produce deformation. After removing the magnetic field, MSMA keeping the same deformation value, the prestressed added to MSMA elements restore its deformation.

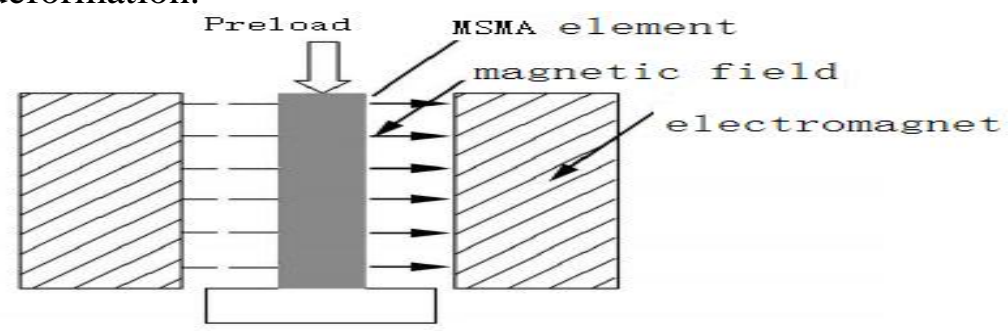

Figure 2. Schematic Diagram of Working Principle of MSMA Linear Displacement Actuator 
Overall Structure Design of MSMA Linear Displacement Actuator. Fig. 3 says the structure principle of MSMA line actuator. the MSMA linear displacement actuator includes its coat, excitation coil, core, MSMA element, the output shaft, the top shaft, the top pressing spring and the adjusting nut and so on, When electromagnet composed by energized core and excitation coil provides magnetic field, MSMA is low permeability material, for improving its work efficiency, the design of the closed magnetic circuit composed by MSMA elements and core structure come into being, because of the high permeability of core, almost all magnetic flux is limited in the iron core, magnetic field lines flow along the path of core, evenly across the MSMA element and perpendicular to the direction of elongation, thus reducing the magnetic leakage of. MSMA came up deformation in the magnetic field, after reducing the magnetic field, it can not automatically return to its original shape, so the spring with a large enough counter force in the direction of elongation of MSMA element, the element returned to shape before extension. In order to control the magnitude of prestressed, is designed adjusting nut to adjust the magnitude of spring's prestressed in spring the upper part. When the excitation coil generates a magnetic field after gaining the current signal, the MSMA element generated elongation change and the elongation effect output to the moving object through the output shaft. The magnetic field disappeared after cutting off the current signal, MSMA element keeps the same shape, the spring pressure to restore its principle's shape, and the actuator's acting effect is completed.

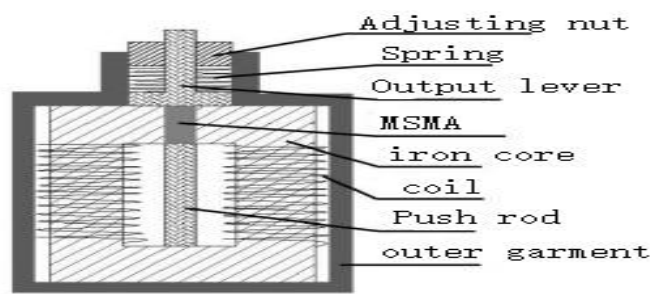

Figure 3. the Overall Structure of the MSMA Linear Displacement Actuator

\section{Conclusion}

MSMA has the features that the fast dynamic response of piezoceramic material and magnetostrictive materials, and the large output strain of temperature control of shape memory alloy, MSMA is expected to become the important material of a new generational drive in the future. This paper expound the deformation mechanism and magnetic control property of the MSMA, design two kinds of MSMA actuator on the basis of these features, and introduce the making principle of MSMA's self-sensingactuator by using inverse magnetic control shape memory, and laid the foundation of MSMA's application in the field of civil engineering. But as a soon appeared new intelligent material, some characteristics of MSMA is not ideal enough, need to be further improvement.

\section{References}

[1] Ou Jinping. Structural vibration control active, semi-active, and intelligent control[M]. Beijing: Science Press, 2003.22-23.[2]

[2] Ullakko Kand Huang J K. Large magnetic-field-induced strains in Ni Mn Ga single crystals[J]. Applied physics letter, 1996, 69:1966- 1968.

[3] Haroon M and Ferri A A. A time and frequency do main approach for identifying nonlinear mechanical system models in absence of an input measurement[J]. J. sound and vibration, 2005, 283(6):1137-1155.

[4] Soderberg. Recent breakthrough development of the magnetic shape memory effect in Ni-Mn-Ga alloys[J]. Smart materials science, 2005:223-235.

[5] Feuch twanger J. Mechanical energy absorption in Ni-Mn-Ga polymer composites[J]. J. of magnetism and magnetic materials, 2004, 272:2038-2049. 
[6] Wilson S A. New materials for micro-scale sensors and actuators: an engineering review[J]. Materials science and engineering R, 2007, 56:1-129.

[7] Takashi $\mathrm{T}$ and MasashiS. Magnetic properties of Fe-Cr-Mn-Si-based ferromagnetic shape memory ribbons[J]. J. of magnetism and magnetic materials, 2007, 310:2776-2778.

[8] Zhang Qingxin magnetic controlled shape memory alloy and basic research on its actuator application [D]. Shenyang University of Technology, 2006:42-50.

[9] UllakkoK, Huang J K, Kokorin V V, et. Magnetic alloy controlled shape memory effection Ni2MnGa intermetallics[J]. ScriPta Ma-terialia, 1997, 36(10):1133-1138.

[10] Massim oPasquale, CarloP.Sasso, Stefano Besseghinietal. Schlagel. Magnetic and mechanical properties of Ni-Mn-Ga single crystals [J]. IEEE Transactions on Magnetics, 2002,38(5):28472849.

[11] TickleR, JamesR D, Shield T etal. Ferro magnetic shape memory effect in the Ni-Mn-Ga system[J]. IEEE Transactions on Magnetics, 1999, 5(5):4301-4310.

[12] Suorsa I, Pagoun is E. Magnetic field-induced stress in the Ni-Mn-Ga magnetic shape memory alloy [J]. Journal of Applied Physics, 2004, 95(9):4958-4961.

[13]Massimo Pasquale, Carlo Paolo Sasso, Stefano Besseghinietal. Field and temperature induced giant strain in single crystal Ni-Mn-Ga [J]. IEEE Transactions on Magnetics, 2001, 37(4):2669-2671.

[14] Oleg Heczko, KariUllakko. Effect of temperature on magnetic properties of Ni-Mn-Ga magnetic shape memory $(\mathrm{msm})$ alloys[J]. IEEE Transactions on Magnetics, 2001, 37(4):2672-2674.

[15] Pakhomov A B, wong C Y, Zhang X X etal. Magnetization and magnetocaloric effect in magnetic shape memory alloys Ni-Mn-Ga[J]. IEEE Transactions on Magnetics, 2001, 37(4):2718- 2720.

[16] James RD, Wuttig M. Magnetostriction of martensite[J]. Philos MagA, 1998,77:1273- 1299.

[17] DeSimone A, James R. A constrained theory of magneto-elasticity [J]. J Mech Phys Solids, 2002, 50: 283- 320. 